\title{
A HYPERSPACE FOR CONVERGENCE SPACES
}

\author{
R. J. GAZIK
}

\begin{abstract}
The purpose of this note is to introduce a convergence structure $h(t)$ on the collection $C(E)$ of nonempty, compact subsets of a Hausdorff convergence space $(E, t)$. It is shown that if $(E, t)$ is topological, then $h(t)$ agrees with the Vietoris topology on $C(E)$. It is proved that $(C(E), h(t))$ is Hausdorff, that it inherits regularity from $(E, t)$ and that it is compact whenever $(E, t)$ is compact and regular.
\end{abstract}

1. Introduction. Let $C(E)$ be the collection of nonempty, compact subsets of a Hausdorff topological space $(E, t)$, and let $v$ be the Vietoris topology on $C(E)$ as a subspace of the collection of nonempty closed subsets of $E$. (See [5] for the definition and properties of $v$.) Using just the definitions, it is easy to see that a filter $\Lambda$ on $C(E)$ converges to $A \in C(E)$ with respect to $v$ if and only if the following are satisfied:

(a) Whenever $A \subset G, G$ open in $E$, then there exists $r \in \Lambda$ such that $U \subset G$ for each $U \in r$.

(b) Whenever $G \cap A \neq \varnothing, G$ open in $E$, then there exists $r \in \Lambda$ such that $G \cap U \neq \varnothing$ for each $U \in r$.

Let us now make two comments. First, we could have started with a notion of convergence of filters in $C(E)$ defined by (a) and (b) above and then recovered the topology $v$ from the convergence. Second, if our object is to study properties of $C(E)$ which can be defined in terms of convergence, then it is immaterial whether we discover $v$ from the convergence or not. In fact, there are numerous important notions of convergence of filters (or nets) which are not induced by any topology. A few examples are (except for special cases): the convergence of closed sets of Choquet (see p. 87 of [2]), continuous convergence of maps from a Hausdorff space to a Hausdorff uniform space (see [3] and its references), the convergence of a net $\left(x_{n}: n \in D\right)$, in a complete lattice, defined by $\lim$ inf $x_{n}=x=\lim \sup x_{n}$. Examples like the ones above led Fischer to formalize the concept of a convergence space in [4]. For the remainder of this paper the reader is assumed to be familiar with the very basic notions of [4].

Received by the editors March 7, 1972.

AMS (MOS) subject classifications (1969). Primary 5410, 5422, 5425.

Key words and phrases. Convergence spaces, convergence structures, limit spaces, limit structures, Vietoris topology, directed sets, ultrafilters, cofinal segments, selections of cofinal segments. 
Our object here is to show that there is a reasonable convergence structure $h(t)$ on the collection of nonempty, compact subsets of an arbitrary Hausdorff convergence space $(E, t)$. That $h(t)$ is reasonable means that it has the properties mentioned in the Abstract.

Filters on a set $E$ will be denoted by Greek letters with two exceptions: The filter of supersets of $b \in E$ is $[b]$ and if $x: D \rightarrow E$ is a net in $E$, the filter of sections of $x$ is $S(x)$. The set of all $x(d), d \geqq e$, is written $S(x, e)$.

If $(E, t)$ is a convergence space and $\alpha$ is a filter on $E, \alpha$ accumulates at $b \in E$ if it is coarser than a filter which $t$-converges to $b$. The filter generated by the $\operatorname{cl}(F, t), F \in \alpha$, is $\operatorname{cl}(\alpha)$. The space $(E, t)$ is regular if $\operatorname{cl}(\alpha) \rightarrow b$ whenever $\alpha \rightarrow b$. See [1] for an equivalent formulation of regularity. $(E, t)$ is compact if each ultrafilter on $E t$-converges and is Hausdorff if no filter $t$-converges to two distinct points.

If $(D(b): b \in B)$ is a set of directed sets, the product set $X(D(b): b \in B)$ is always directed by the product order. That is, $f \geqq g$ if and only if $f(b) \geqq$ $g(b)$ for each $b \in B$. Furthermore, if $\alpha$ is a filter on a set $E$ then $\alpha$ will be considered as a directed set with $F \geqq G$ if and only if $F \subset G, F, G \in \alpha$. These two conventions will be used without explicit mention.

2. Definition of $h(t)$. Let $C(E)$ be the collection of nonempty compact subsets of a convergence space $(E, t)$. If $\Lambda$ is a filter on $C(E)$, a cofinal segment of $\Lambda$ is a pair $(D, f)$, where $D$ is a directed set, $f: D \rightarrow \Lambda$ is a function, and for each $r \in \Lambda$ there exists $d \in D$ such that $f(e) \subset r$ whenever $e \geqq d$. If $(D, f)$ is a cofinal segment of $\Lambda$, then a selection of $(D, f)$ is a pair $(x, g)$, where $x: D \rightarrow E, g: D \rightarrow C(E)$ are functions and $x(d) \in g(d), g(d) \in$ $f(d)$ for all $d \in D$.

Definition 2.1. Let $C(E)$ be the collection of nonempty compact subsets of a Hausdorff convergence space $(E, t)$. Let $\Lambda$ be a filter on $C(E)$ and $A \in C(E)$. Then $\Lambda h(t)$-converges to $A$ (written $\Lambda \rightarrow A$ ) if and only if the following are satisfied:

(1) For each cofinal segment $(D, f)$ of $\Lambda$ and each selection $(x, g)$ of $(D, f)$, the section filter $S(x)$ accumulates at some point of $A$.

(2) For each $a \in A$ there is some filter $\alpha$, with $\alpha \rightarrow a$, and some cofinal segment $(D, f)$ of $\Lambda$ with the following property: For each $F \in \alpha$ there exists $d \in D$ such that, whenever $e \geqq d$ and $U \in f(e)$ then $F \cap U \neq \varnothing$.

The reader should compare Definition 2.1 with the Vietoris convergence of $\S 1$ and see what alterations have been made in the convergence space case. That $h(t)$ is indeed a convergence structure on $C(E)$ is shown below.

THEOREM 2.1. If $C(E)$ is the collection of nonempty, compact subsets of a Hausdorff convergence space $(E, t)$, then $h(t)$ is a convergence structure on $C(E)$. 
Proof. If $A \in C(E)$ then $[A] \rightarrow A$ because of the following:

(1) If $(x, g)$ is a selection of a cofinal segment $(D, f)$ of $[A]$, then $x(d) \in g(d)=A$ for $d$ sufficiently large. This means that $A$ contains some $S(x, d)$. The compactness of $A$ now shows that $S(x)$ accumulates at some point of $A$.

(2) If $a \in A$ define $\alpha=[a]$ and, for $r \in[A]$, put $f(r)=r$. It is clear that $([A], f)$ is a cofinal segment of $[A]$ and that $\alpha$ and $([A], f)$ satisfy $(2)$ of Definition 2.1 for $[A]$.

Suppose now that $\Lambda \rightarrow A$ and $\Psi \geqq \Lambda$. We show that $\Psi \rightarrow A$.

(1) Let $(x, g)$ be a selection of a cofinal segment $(D, f)$ of $\Psi$. On $D \times \Lambda$ define functions as follows: $F(d, r)=f(d) \cup r, G(d, r)=g(d), X(d, r)=x(d)$. Then $(D \times \Lambda, F)$ is a cofinal segment of $\Lambda$ and $(X, G)$ is a selection of $(D \times \Lambda, F)$. Thus $S(X)$ accumulates at some point of $A$. But $S(x) \leqq S(X)$ so $S(x)$ accumulates at a point of $A$.

(2) Let $a \in A$ and let $\alpha,(D, f)$, be the filter and cofinal segment which satisfy (2) of Definition 2.1 for $\Lambda$. On $D \times \Psi$ define $F(d, r)=f(d) \cap r$. It is clear that $\alpha$ and $(D \times \Psi, F)$ satisfy (2) of Definition 2.1 for $\Psi$.

Finally, suppose $\Phi, \Psi h(t)$-converge to $A$. We prove that $\Phi \wedge \Psi^{\circ} \rightarrow A$.

(1) Let $(x, g)$ be a selection of a cofinal segment $(D, f)$ of $\Phi \wedge \Psi$. Let $K$ be any well ordered, cofinal subset of $D$. It is asserted that $B(K)=$ $(g(d): d \in K)$ is in some ultrafilter finer than $\Phi \wedge \Psi$. If this is not so, $C(E)-$ $B(K)$ is in each ultrafilter finer than $\Phi \wedge \Psi$ and hence in $\Phi \wedge \Psi$. The properties of $(D, f), K$ now yield the contradiction that $g(d) \in C(E)-B(K)$ for some $d \in K$.

Let $\Lambda(K)$ be an ultrafilter finer than $\Phi \wedge \Psi$ which contains $B(K)$.

Case 1. For some $K, \Lambda(K)$ is not a $[g(d)], d \in K$. Then, by transfinite induction, $\Lambda(K)$ contains $B(K, d)=(g(e): e \geqq d, e \in K)$ for each $d \in K$. On $K \times \Lambda(K)$ define functions as follows: $F(d, r)=B(K, d) \cap r ; G(d, r)=g(p)$ for some $p \geqq d, p \in K$, with $g(p) \in B(d) \cap r ; X(d, r)=x(p)$. ( $G$ and $X$ exist by the axiom of choice.) It is clear that $(K \times \Lambda(K), F)$ is a cofinal segment of $\Lambda(K)$ and that $(X, G)$ is a selection of $(K \times \Lambda(K), F)$. Now $\Lambda(K)$ is an ultrafilter so it is finer than one of $\Phi, \Psi$. Whence $\Lambda(K) \rightarrow A$ and $S(X)$ accumulates at some point of $A$. From $S(x) \leqq S(X)$ it follows that $S(x)$ accumulates at some point of $A$.

Case 2. For each well ordered, cofinal subset $K$ of $D, \Lambda(K)$ is some $[g(d(K))], d(K) \in K$.Then $[g(d(K))]$ is finer than one of $\Phi, \Psi$ and $[g(d(K))] \rightarrow$ $g(d(K))$. Thus, for each $K, g(d(K))=A$ and $[A]=\Lambda(K)$. But, if $K$ is one well ordered, cofinal subset of $D$ then $K(d)=(e \in K: e \geqq d)$ is another for each $d \in K$. Then the set $(g(e): e \geqq d, e \in K) \in \Lambda(K(d))=[A]$ and, from this, the set $M=(d \in K: g(d)=A)$ is cofinal in $K$. Hence $(x(d): d \in M) \subset A$ so, since $A$ is compact, $S(x)$ accumulates at a point of $A$. It has been shown that (1) of Definition 2.1 holds for $\Phi \wedge \Psi$ and $A$. 
(2) Let $a \in A$. By assumption there are filters $\alpha, \beta$ which $t$-converge to $a$, and cofinal segments $(f, D),(p, H)$ of $\Phi, \Psi$ respectively which satisfy (2) of Definition 2.1. Put $\gamma=\alpha \wedge \beta$ and $F(d, h)=f(d) \cup p(h)$. Then $\gamma,(D \times H, F)$, satisfy (2) of Definition 2.1 for $\Phi \wedge \Psi$ and $A$. This completes the proof.

Next it will be shown that $h(t)$ indeed is a generalization of the Vietoris topology.

THEOREM 2.2. If $C(E)$ is the set of nonempty, compact subsets of a Hausdorff topological space $(E, t)$, then $h(t)$ is the Vietoris topology on $C(E)$.

Proof. Suppose that $\Lambda v$-converges to $A \in C(E)$. Let $(x, g)$ be a selection of a cofinal segment $(D, f)$ of $\Lambda$. Suppose $S(x)$ fails to accumulate at any point of $A$. A standard compactness argument shows that there exists a finite subset $T$ of $A$, points $d(a) \in D$, and open sets $G(a)$ containing $a$, such that $A \subset \bigcup(G(a): a \in T)$ and $B=\bigcap(S(x, d(a)): a \in T) \cap$ $\bigcup(G(a): a \in T)=\varnothing$. By (a) of $\S 1$ there exists $r \in \Lambda$ such that $U \subset$ $\bigcup(G(a): a \in T)$ for each $U \in r$. But $(D, f)$ is a cofinal segment of $\Lambda$ so $g(d) \in f(d) \subset r$ for $d$ sufficiently large. Hence, if $d \geqq d(a)$ for all $a \in T$ then $x(d) \in B$, a contradiction. It follows that (1) of Definition 2.1 holds for $\Lambda$ and $A$.

To prove that (2) holds let $a \in A$. Let $N$ be the collection of open subsets of $E$ which contain $a$. Order $N$ by reverse inclusion. If $(r, G) \in \Lambda \times N$ then, by (b) of $\S 1$, there is an $f(r, G) \subset r$ with $U \cap G \neq \varnothing$ for $U \in f(r, G)$. Define $\alpha$ to be the neighborhood filter at $a$. Then $\alpha,(\Lambda \times N, f)$, satisfy (2) for $\Lambda$ and $A$. We have shown that $\Lambda \rightarrow A$ whenever $\Lambda v$-converges to $A$.

Conversely, let $\Lambda \rightarrow A$. Suppose (a) of $\S 1$ fails for $\Lambda$. Then there is an open set $G, A \subset G$, such that $r \in \Lambda$ implies there exists $g(r) \in r$ for which $g(r) \notin G$. If $f$ is the identity map on $\Lambda$ and $x(r) \in g(r)-G$ then $(x, g)$ is a selection of the cofinal segment $(\Lambda, f)$. By assumption $S(x)$ accumulates at some $b \in A$. But each $S(x, r) \subset E-G, E-G$ closed, so $b \in E-G \subset E-A$ which is a contradiction. It has been shown that (a) of $\S 1$ holds for $\Lambda$. To prove that (b) holds suppose $G \cap A \neq \varnothing, G$ open. Let $a \in G \cap A$. There is some filter $\gamma \rightarrow a$ and some cofinal segment $(D, f)$ of $\Lambda$ which satisfy (2) of Definition 2.1 for $\Lambda$. Moreover $\gamma$ is finer than the neighborhood filter at the point $a$ since $t$ is topological. Thus $G \in \gamma$ and $U \cap G \neq \varnothing$ for $U \in f(d)$ and some $d \in D$.

\section{Separation properties of $h(t)$.}

THEOREM 3.1. Let $(E, t)$ be a Hausdorff convergence space. Then $(C(E), h(t))$ is Hausdorff.

Proof. Assume $\Lambda \rightarrow A$ and $\Lambda \rightarrow B$. Let $a \in A$ and let $\alpha,(D, f)$ be the filter and cofinal segment of $\Lambda$ which satisfy (2) of Definition 2.1 for $\Lambda$ and 
$A$. If $H \in \alpha$ there exists $d(H) \in D$ such that whenever $d \geqq d(H)$ and $U \in f(d)$ then $U \cap H \neq \varnothing$. Further, if $(d, H) \in D \times \alpha$ there exists $p(d, H) \geqq d(H), d$. Now there are functions on $D \times \alpha$ with the following properties: $F(d, H)=$ $f(p(d, H)), G(d, H)$ is arbitrary in $F(d, H), X(d, H) \in G(d, H) \cap H$. Then $(X, G)$ is a selection of the cofinal segment $(D \times \alpha, F)$ of $\Lambda$ so $S(X)$ accumulates at some point of $B$. However $\alpha \leqq S(X)$ so $\alpha \rightarrow a \in A$ and $\alpha$ accumulates at some point of $B$. Since $(E, t)$ is Hausdorff, $a \in B$. It has been shown that $A \subset B$; similarly $B \subset A$. So $A=B$ and $(C(E), h(t))$ is Hausdorff.

In order to show that $C(E)$ inherits regularity from $E$ two lemmas will be employed. The reader will recognize the second of these as the analogue of a "diagonal net" theorem from topology.

LEMMA 3.2. Let $(E, t)$ be a Hausdorff convergence space. If $F \subset E$, $r \subset C(E)$ and $U \cap F \neq \varnothing$ for each $U \in r$, then $U \cap \operatorname{cl}(F, t) \neq \varnothing$ for each $U \in \operatorname{cl}(r, h(t))$.

Proof. If $U \in \operatorname{cl}(r, h(t))$, there is a filter $\Lambda$ on $C(E)$ with $r \in \Lambda$ and $\Lambda \rightarrow U$. Define functions on $\Lambda$ as follows: $f(s)=s, g(s)$ is arbitrary in $s \cap r$ and $x(s) \in g(s) \cap F$. Then $S(x)$ accumulates at some point $b \in U$. But $F \in S(x)$ so $b \in \operatorname{cl}(F, t)$. Thus $b \in U \cap \operatorname{ncl}(F, t)$.

LEMMA 3.3. Let $(E, t)$ be a regular, Hausdorff convergence space. Let $D$ be a directed set and suppose there are filters $\alpha(d)$ on $E$ with $\alpha(d) \rightarrow x(d)$ for each $d \in D$. Define $I=D \times X(\alpha(d): d \in D)$ and suppose $X: I \rightarrow E$ has the property that $X(d, h) \in h(d)$ for each $h$ and each $d$. Then, if $S(X)$ accumulates at $y \in E$ so does $S(x)$.

Proof. Suppose $S(X)$ accumulates at $y$. Then $S(X) \leqq \beta \rightarrow y$ for some filter $\beta$. Since $(E, t)$ is regular, $S(x)$ accumulates at $y$ if $\sup (S(x), \operatorname{cl}(\beta))$ exists. Let $S(x, d)$ be an arbitrary section of $S(x)$ and suppose $B \in \beta$. If, for some $p \geqq d, B \cap H \neq \varnothing$ for all $H \in \alpha(p)$ then the filter generated by the $B \cap H, H \in \alpha(p)$, contains $B$ and is finer than $\alpha(p)$. Since $\alpha(p) \rightarrow x(p)$, this means $x(p)$ is in $\operatorname{cl}(B) \cap S(x, d)$. Hence $\operatorname{cl}(B) \cap S(x, d) \neq \varnothing, \sup (S(x), \operatorname{cl}(\beta))$ exists, and $S(x)$ accumulates at $y$. Suppose, contrariwise, that for each $p \geqq d$ there exists $H(p) \in \alpha(p)$ with $H(p) \cap B=\varnothing$. Define a function $h$ by putting $h(p)=H(p)$ if $p \geqq d$ and $h(p)=E$ otherwise. Since $X(d, h) \in h(d)$, it follows that $S(X,(d, h)) \subset E-B$. Then $E-B \in \beta$ which contradicts $B \in \beta$.

THEOREM 3.4. Let $(E, t)$ be a regular Hausdorff convergence space. Then $(C(E), h(t))$ is regular.

Proof. Suppose $\Lambda \rightarrow A$ and let $(x, g)$ be a selection of the cofinal segment $(D, f)$ of $\operatorname{cl}(\Lambda)$. Then, for each $(d, r) \in D \times \Lambda$ there exists $n(d, r) \geqq$ $d$ for which $f(n(d, r)) \subset \operatorname{cl}(r, h(t))$. Thus, there are filters $\Psi(d, r)$ on $C(E)$ 
with $r \in \Psi(d, r)$ and $\Psi(d, r) \rightarrow g(n(d, r))$. Since $x(n(d, r)) \in g(n(d, r))$ it follows that there are filters $\alpha(d, r) \rightarrow x(n(d, r))$ and cofinal segments $(D(d, r), j(d, r))$ of $\Psi(d, r)$ which satisfy (2) of Definition 2.1 for $\Psi(d, r)$ and $g(n(d, r))$. This and the fact that $r \in \Psi(d, r)$ shows that there exist functions defined on $I=(D \times \Lambda) \times X(\alpha(d, r):(d, r) \in D \times \Lambda)$ as follows: $F((d, r), h)=r, G((d, r), h) \in r$ with $G((d, r), h) \cap h(d, r) \neq \varnothing, X((d, r), h) \in$ $G((d, r), h) \cap h(d, r)$. Now $(X, G)$ is a selection of the cofinal segment $(I, F)$ of $\Lambda$ so $S(X)$ accumulates at a point $y \in A$. By Lemma 3.3, $S(x n)$ accumulates at $y$ and it is clear, then, that $S(x)$ accumulates at $y \in A$. Thus (1) of Definition 2.1 holds for $\operatorname{cl}(\Lambda)$ and $A$.

Next, let $a \in A$ and let $\alpha, \alpha \rightarrow a,(D, f)$, be the filter and final segment of $\Lambda$ which satisfy (2) of Definition 2.1 for $\Lambda$ and $A$. Define $m: D \rightarrow \operatorname{cl}(\Lambda)$ by $m(d)=\operatorname{cl}(f(d), h(t))$. Since $(E, t)$ is regular, $\operatorname{cl}(\alpha) \rightarrow a$ and, by Lemma 3.2, $(D, m)$ and $\operatorname{cl}(\alpha)$ satisfy $(2)$ of Definition 2.1 for $\operatorname{cl}(\Lambda)$ and $A$.

It has been demonstrated that $\operatorname{cl}(\Lambda) \rightarrow A$ whenever $\Lambda \rightarrow A$ so $(C(E), h(t))$ is regular and the proof is complete.

LEMMA 3.5. Let $(x, g)$ be a selection of a cofinal segment $(D, f)$ of a filter $\Lambda$ on $C(E)$ and suppose $S(x)$ accumulates at $a$ point $b \in E$. Then, there is a selection $(X, G)$ of a cofinal segment $(I, F)$ of $\Lambda$ with $S(X) \rightarrow b$.

Proof. By assumption $S(x) \leqq \alpha \rightarrow b$ and we may define functions on $I=D \times \alpha$ as follows: $F(d, A)=f(n(d, A))$ where $n(d, A) \geqq d$ and $x(n(d, A)) \in$ $A ; G(d, A)=g(n(d, A)) ; X(d, A)=x(n(d, A))$. It follows easily that $(X, G)$ is a selection of $(I, F)$ and that $S(X) \rightarrow b$.

LEMMA 3.6. Let $\Lambda$ be a filter on $C(E)$ with $(E, t)$ compact, regular and Hausdorff. Let $A$ be the set of all $z$ for which there exists a selection $(x, g)$ of a cofinal segment of $\Lambda$ such that $S(x)$ accumulates at $z$. Then $A$ is a compact subset of $E$.

Proof. Since $(E, t)$ is compact, Hausdorff, it suffices to prove that $\operatorname{cl}(A, t) \subset A$. Toward this end let $b \in \operatorname{cl}(A, t)$. There is a filter $\alpha \rightarrow b$ with $A \in \alpha$. So, for each $H \in \alpha$ there exists $y(H) \in H \cap A$. By definition of $A$ and lemma 3.5 there are selections $(x(H), g(H))$ of cofinal segments $(D(H), f(H))$ with $S(x(H)) \rightarrow y(H)$. Put $I=\alpha \times X(D(H): H \in \alpha)$ and define functions on $I$ as follows: $F(H, p)=f(H)(p(H)), G(H, p)=g(H)(p(H))$, $X(H, p)=x(H)(p(H))$. Then $(X, G)$ is a selection of the cofinal segment $(I, F)$. Since $(E, t)$ is compact $S(X) \leqq \beta \rightarrow z$ for some $z \in E$. Since $(E, t)$ is regular, an argument similar to the one given in the first part of Theorem 3.4 shows that $S(y)$ accumulates at $z$. But surely $S(y) \rightarrow b$ so $z=b$. Thus $S(X)$ accumulates at $b$ and $b \in A$ by definition of $A$.

THEOREM 3.7. Let $(E, t)$ be a compact, Hausdorff, regular convergence space. Then $(C(E), h(t))$ is compact. 
Proof. Let $\Lambda$ be an ultrafilter on $C(E)$. Define $A$ to be the set of all points $z$ for which there exists a selection $(x, g)$ of a cofinal segment of $\Lambda$ such that $S(x)$ accumulates at $z$. By Lemma 3.6, $A \in C(E)$. We prove that $\Lambda \rightarrow A$.

(1) That (1) of Definition 2.1 is satisfied for $\Lambda$ and $A$ is clear from the definition of $A$ and the compactness of $(E, t)$.

(2) Let $a \in A$. From definition and Lemma 3.5 there is some selection $(x, g)$ of some cofinal segment $(D, f)$ of $\Lambda$ with $S(x) \rightarrow a$. If $S(x, d)$ is an arbitrary generator of $S(x)$ define $r$ to be the set of all $B \in C(E)$ such that $B \cap S(x, d) \neq \varnothing$. Since $\Lambda$ is an ultrafilter either $r \in \Lambda$ or $C(E)-r \in \Lambda$. But $C(E)-r \in \Lambda$ leads to $f(e) \subset C(E)-r$ for $e$ sufficiently large. This in turn leads to the contradiction $g(e) \cap S(x, d)=\varnothing$ for $e$ sufficiently large. Thus $r \in \Lambda$ and it follows that $(D, f)$ and $S(x)$ satisfy (2) of Definition 2.1 for $\Lambda$ and $A$.

The author wishes to thank the referee for several very helpful suggestions.

\section{REFERENCES}

1. H. J. Biesterfeldt, Jr., Regular convergence spaces, Nederl. Akad. Wetensch. Proc. Ser. A 69=Indag. Math. 28 (1966), 605-607. MR 34 \#5048.

2. G. Choquet, Convergences, Ann. Univ. Grenoble Sect. Sci. Math. Phys. 23 (1948), 57-112. MR 10, 53.

3. C. H. Cook and H. R. Fischer, Uniform convergence structures, Math. Ann. 173 (1967), 290-306. MR 36 \#845.

4. H. R. Fischer, Limesröume, Math. Ann. 137 (1959), 269-303. MR 22 \#225.

5. E. A. Michael, Topologies on spaces of subsets, Trans. Amer. Math. Soc. 71 (1951), 152-182. MR 13, 54.

Department of Mathematics, Arkansas State University, State University, ARKANSAS 72467 